\title{
HBV upregulates AP-1 complex subunit mu-1 expression via the JNK pathway to promote proliferation of liver cancer cells
}

\author{
YANBO KOU $^{1 *}$, XIAOQING YAN $^{2 *}$, QINGYA LIU $^{1}$, XIAO WEI $^{1}$, BO ZHANG $^{1}$, XIANGYANG LI $^{1}$, \\ WEI PAN $^{1}$, FANYUN KONG ${ }^{1}$, YUGANG WANG ${ }^{1}$, KUIYANG ZHENG ${ }^{1}$ and RENXIAN TANG ${ }^{1}$ \\ ${ }^{1}$ Jiangsu Key Laboratory of Immunity and Metabolism, Department of Pathogenic Biology and Immunology, \\ Xuzhou Medical University; ${ }^{2}$ Institute of Emergency and Rescue Medicine, Laboratory of Emergency Medicine, \\ Xuzhou Medical University, Xuzhou, Jiangsu 221004, P.R. China
}

Received September 3, 2018; Accepted April 5, 2019

DOI: $10.3892 / \mathrm{ol} .2019 .10291$

\begin{abstract}
Although hepatitis B virus (HBV) infection is responsible for liver cancer, the exact mechanism of its action remains unclear. $\mu 1$ adaptin is an intrinsic part of the clathrin adaptor AP-1 complex. In addition to its canonical biological function that involves cargo sorting and vesicular transport, recent studies have demonstrated that $\mu 1$ adaptin participates in cell growth and proliferation. The aim of the present study was to investigate the effects of the clathrin adaptor AP-1 complex subunit mu-1 (AP1M1) on liver cancer cell proliferation. The present study reports for the first time that AP1M1 is upregulated in the HBV-transfected HepG2.215 liver cancer cells. Silencing of AP1M1 in HepG2.215 cells suppressed their proliferation, while the overexpression of AP1M1 in HepG2 cells promoted cell proliferation. The data suggested that AP1M1 is one of the crucial factors involved in the progression of liver cancer caused by HBV infection. In addition, it was demonstrated that HBV facilitated AP1M1 expression in a JNK-dependent manner. The increased expression levels of AP1M1 enhanced phosphorylation of protein kinase B and accelerated cell proliferation. Unraveling the effects of AP1M1 on liver cancer cell proliferation and the mechanism of AP1M1 transcriptional regulation may provide new therapeutic targets for HBV-positive liver cancer.
\end{abstract}

Correspondence to: Professor Renxian Tang or Professor Kuiyang Zheng, Jiangsu Key Laboratory of Immunity and Metabolism, Department of Pathogenic Biology and Immunology, Xuzhou Medical University, 209 Tongshan Road, Xuzhou, Jiangsu 221004, P.R. China

E-mail: tangrenxian-t@163.com

E-mail: zky02@163.com

${ }^{*}$ Contributed equally

Key words: hepatitis B virus, AP1M1, liver cancer, cell proliferation

\section{Introduction}

Liver cancer is one of the common malignant tumors worldwide that is associated with very poor prognosis. A variety of factors contribute to liver cancer development, such as age, gender, obesity, diabetes and chronic hepatitis B virus (HBV) infection. Among the different factors that affect liver cancer progression, HBV infection exhibits the highest proportion and according to epidemiological data it accounts for $53 \%$ of all liver cancers in China (1). HBV is a hepadnavirus. The overlapping open reading frames of $\mathrm{C}, \mathrm{P}, \mathrm{S}$ and $\mathrm{X}$ are responsible for the genome assembly of HBV (2,3). Previous studies have demonstrated that HBV participates in several cellular processes including proliferation, migration, invasion and apoptosis that are associated with liver cancer progression (4-7). The dominant theory suggests that HBV proteins, notably HBX, disturb the expression of proto-oncogenes or tumor suppressor genes resulting in the progression of liver cancer. However, the mechanism of this process remains unclear (8).

Accumulating evidence suggests that HBX contributes to liver cancer development by direct and indirect mechanisms. Nuclear factor- $\kappa \mathrm{B}(\mathrm{NF}-\kappa \mathrm{B})$ and activator protein 1 (AP-1) can be activated by HBX that subsequently promotes cell proliferation $(9,10)$. HBX can also interact with different oncoproteins, such as Src and Ras to promote cell proliferation $(11,12)$.

The clathrin adaptor AP-1 complex is a primary regulator of vesicular transport and protein sorting. An integrated AP-1 complex comprises four subunits termed $\beta 1, \gamma, \delta 1$ and $\mu 1$. The major function of the $\mu 1$ and $\beta 1$ subunits involves the binding of transmembrane proteins by their sorting motifs, while $\gamma$ and $\delta 1$ interact with clathrin and link transmembrane proteins with this protein $(13,14) . \mu 1 \mathrm{~A}$ and $\mu 1 \mathrm{~B}$ adaptin are two representative isoforms of the $\mu 1$ subunit that exhibit distinct expression (15). $\mu 1 \mathrm{~B}$ is mainly expressed in polarized epithelial cells compared with $\mu 1 \mathrm{~A}$ adaptin, which is ubiquitously expressed. Previous studies indicated that the $\mu 1$ subunit binding motif ( YXXФ) of the C-terminal region of the transmembrane proteins regulates their sorting or vesicular transport (16-18). In addition, recent studies have demonstrated new functions of the $\mu 1$ subunit in viral invasion, growth, cell proliferation and hyperplasia by its interaction with related transmembrane proteins (19-22). However, whether adaptin 
further participates in liver cancer development remains unknown.

The present study, aimed to investigate the effects of AP1M1 on liver cancer cell proliferation and to explore the associated mechanism of this process. It is reported that the $\mu 1 \mathrm{~A}$ adaptin AP1M1 is upregulated in liver cancer cells compared with the corresponding expression in normal hepatocytes. HBV facilitated AP1M1 expression in a c-Jun $\mathrm{N}$-terminal kinase (JNK)-dependent manner. Overexpression of AP1M1 in HepG2 cells promoted its proliferation via the protein kinase B (AKT) pathway, while AP1M1 silencing suppressed proliferation of HBV-transfected HepG2 cells.

\section{Materials and methods}

Cell lines and reagents. The human non-tumor hepatic cell L02 and liver cancer cell lines HepG2 and Huh7 were obtained from the Cell Bank of the Chinese Academy of Sciences (Shanghai, China). HBV genome (pUC18-HBV1.2) transfected HepG2 (HepG2.215) was from the Peking University Hepatology Institute (Beijing, China). HBX deficiency HepG2.215 (HepG2.215 $\Delta$ HBX) and HBX overexpression HepG2 (HepG2-HBX) were obtained as previously described and expression of HBX in the cells mentioned above was detected $(23,24)$. The cells were cultured in Dulbecco's Modified Eagle Medium (high glucose) (HyClone; GE Healthcare Life Sciences, Logan UT, USA) supplemented with penicillin-streptomycin and $10 \%$ fetal bovine serum (Serana Europe GmBh, Pessin, Germany) at $37^{\circ} \mathrm{C}$ in $5 \% \mathrm{CO}_{2}$. For AKT, JNK or nuclear factor $(\mathrm{NF}) \kappa \mathrm{B}$ inhibitor treatment, $10 \mathrm{mM}$ LY294002 (Sigma-Aldrich, Merck KGaA), 10 mM SP600125 (Sigma-Aldrich, Merck KGaA) and 5 mM BAY11-7082 (Sigma-Aldrich; Merck KGaA) in DMSO were administrated to the cells with a final concentration of $10 \mu \mathrm{M} \mathrm{LY294002,}$ $10 \mu \mathrm{M}$ SP600125 and $5 \mu \mathrm{M}$ BAY11-7082 respectively in $37^{\circ} \mathrm{C}$ and $5 \% \mathrm{CO}_{2}$ atmosphere. After $24 \mathrm{~h}$, the cells were washed twice with PBS and collected for subsequent detection. In the cell viability assay, LY294002 was administrated immediately after cell adherence and maintained to the end.

Plasmids. For APIM1 overexpression plasmid construction, the human $A P 1 M 1$ open reading frame was amplified from cDNAs extracted from HepG2 with the following primers 5'-CTA $\underline{G C T}$ AGCATGTCCGCCAGCGCCGTCTAC-3', 5'-CCGGAATTC TCACTGGGTCCGGAGCTGGTAATCT-3' and cloned into eukaryotic vector pcDNA3. $1^{(+)}$by restriction enzymes NheI and EcoRI. For aplml silencing, the small interfering (si)RNAs against AP1M1 (siRNA1, 5'-GCTATCACGCTTCGAGAA TGA-3' and siRNA2: 5'-GGCATCAAGTATCGGAAGA-3') were administrated to HepG2.215. For HBX overexpression plasmid construction, the full length HBX sequence was amplified from the HBV genome with the following primers 5'-TGT G $\underline{A A G C T} \underline{T A T G G C T G C T A G G C-3 ', 5 '-T G T G G A A T T C T T}$ AGGCAGAGGTG-3' and cloned into pcDNA3.1 ${ }^{(+)}$by restriction enzymes HindIII and EcoRI. The pUC18-HBV1.2- $\triangle \mathrm{HBX}$ plasmid, a vector containing a synthetic HBV mutant with a deleted HBX gene, was constructed in Transheep Bio (Shanghai, China, http://www.transheep.com/) and was used for HepG2.215 $\Delta \mathrm{HBX}$ construction. The transfection was done using Lipo2000 (Thermo Fisher Scientific, Inc., Waltham,
USA) according to the manufactory's protocol. The APIMI overexpression liver cancer cell HepG2-OEAP1M1, control HepG2-NC were obtained after screening with $500 \mu \mathrm{g} / \mathrm{ml} \mathrm{G} 418$.

Western blotting. Cells were homogenized in RIPA lysis buffer (Beyotime Institute of Biotechnology, Shanghai, China) containing with freshly added protease inhibitors and phosphatase inhibitor. Protein was analyzed with a bicinchoninic acid assay (Beyotime Biotechnology, Shanghai, China) to quantify protein concentration. A total of $30 \mu \mathrm{g}$ of total protein was loaded per lane. Proteins were resolved by $10 \%$ SDS-PAGE and transferred to PVDF membranes. 3\% BSA (Solarbio, Beijing, China) was administrated at room temperature for 90 min to block nonspecific binding sites. The anti-AP1M1 rabbit polyclonal antibodies (ab111135) was purchased from Abcam (Cambridge, UK), the $\beta$-actin antibody (AC026) was purchased from Abclonal (Wuhan China), the antibodies for total JNK (\#9252), AKT (\#4691) and phosphorylated JNK (\#4668) and AKT (\#4060) were purchased from CST (Boston, MA, USA). The final dilution of AP1M1 antibodies was 1:500, $\beta$-actin antibody was 1:5,000, the others were 1:1,000. After incubation with the primary antibodies overnight at $4^{\circ} \mathrm{C}$, the PVDF membranes were washed with TBST buffer thrice and incubated with 1:1,000 diluted HRP-linked secondary antibody (\#7074) at room temperature for $60 \mathrm{~min}$. The labeled bands were detected with ECL Plus Western blotting detection kit (Beyotime Institute of Biotechnology).

Reverse transcription-quantitative PCR (RT-qPCR). Total RNA was extracted from cells homogenized in TRIzol (Thermo Fisher Scientific, Inc.; 3 replica wells for each cell line). A total of $1 \mu \mathrm{g}$ of purified RNA was used for reverse transcription with a High-Capacity cDNA Reverse Transcription kit (Takara, Bio, Inc., Otsu, Japan) at $37^{\circ} \mathrm{C}$ for $15 \mathrm{~min}$. The resulting cDNA was used for quantitative PCR on a real-time PCR detection system (Roche LightCycler480) with two-step method. The cycling conditions were: Initial denaturation $95^{\circ} \mathrm{C}$ for $120 \mathrm{sec}$, then 40 cycles of $95^{\circ} \mathrm{C}$ for $10 \mathrm{sec}, 60^{\circ} \mathrm{C}$ for $20 \mathrm{sec}$. Relative mRNA expression level was determined using the $2^{-\Delta \Delta \mathrm{Cq}}$ method with $\beta$-actin as the internal reference control (25). The sequences of primers used were as following: APIMI (5'-GACCGCACCATC TCCTTCATCC-3', 5'-TTGACCGCCGCTTGAACTGG-3'); $\beta$-actin (5'GGCATCGTGATGGACTCCG3', 5'-GCTGGA AGGTGGACAGCGA-3'); HBX (5'-GTGAAGCTTATGGCT GCTAGGC-3', 5'-GTGGAATTCTTAGGCAGAGGTG-3').

Cell viability assay. A density of $2 \times 10^{4}$ cells $/ \mathrm{ml}$ cells were prepared and $100 \mu \mathrm{l}$ of cell suspension was placed into 96 -well plates ( 6 replica wells for each cell line). The number of viable cells was determined by Cell Counting Kit-8 (CCK-8; Dojindo Molecular Technologies, Inc., Kumamoto, Japan) according to the manufacturer's protocol.

Colony formation assay. A total of 200 cells of each cell line were added to 6-well culture plate with three paralleled repeats. After incubation at $37^{\circ} \mathrm{C}$ in $5 \% \mathrm{CO}_{2}$ for two weeks, the cells were washed with PBS and stained with Crystal Violet Staining Solution for $10 \mathrm{~min}$ at room temperature. The number of colonies containing at least 30 cells was counted under a white light microscope at a magnification of $\mathrm{x} 100$. 
Prediction of transcription factors binding sites on promoter. The 2,000 bp sequences upstream of AP1MI transcription start site was used to predict the key transcription factors of regulating AP1M1 expression with online software (http://jaspar. binf.ku.dk/).

Dual luciferase assay. The APIM1 promoter $(2,000 \mathrm{bp}$ upstream of transcription start site) was cloned into the multiple cloning sites of pGL3-basic reporter vector (Promega Corporation, Madison, WI, USA). A total of $1 \times 10^{5}$ cells of each cell line were seeded into 12-well plates with three paralleled repeats and co-transfected with reporter plasmid (encoding firefly luciferase) and internal reference control vector (encoding renilla luciferase) using Lipofectamine 2000 (Thermo Fisher Scientific, Inc., Waltham, USA). After 48 h, luciferase assay was carried out using the Dual Luciferase Assay kit (Promega Corporation) following the manufacturer's protocol. The renilla luciferase activity was used for normalization. The relative luciferase activity of HepG2 transfected with pGL3-APIM1 promoter was defined as $100 \%$.

Public expression profile data analysis. The 27 expression profile data of acute liver failure patients (GDS4387) uploaded by Nissim et al (26) were cited from NCBI GEO Profiles database (https://www.ncbi.nlm.nih.gov/geoprofiles/85359804). Among all the 27 samples, 10 samples were HBV-negative while 17 were HBV-positive. Value of APIMI transcription was analysed by Prism 5.0 (GraphPad Software, Inc., La Jolla, CA, USA) software.

Statistical analysis. Data were analyzed using Prism (GraphPad Software, Inc.) and are presented as the mean \pm standard error of the mean. Statistical significance was determined using the unpaired two-tailed Student's $t$ test for single variables and two-way analysis of variance followed by Bonferroni posttests for multiple variables. $\mathrm{P}<0.05$ was considered to indicate a statistically significant difference.

\section{Results}

HBV upregulates APIM1 expression in liver cancer cells. In the authors' recent study, an Affymetrix GeneChip Human Gene 1.0 ST array was performed to investigate the role of HBV in the genetic alteration of liver cancer cells (23). In addition to interleukin-7R, it was reported previously that the transcription of the AP-1 complex subunit mu-1 coding gene $A P 1 M 1$ was also upregulated in pUC18-HBV1.2 transfected liver cancer cells. To further determine whether AP1M1 was upregulated in HBV-positive liver cancer cells, the transcriptional levels of APIM1 in human hepatocyte lineage L02 cells and in Huh7, HepG2 and HepG2.215 liver cancer cells (stable transfection with the HBV genome) were initially tested. Liver cancer cells expressed high levels of AP1M1, while hepatocytes expressed relatively lower levels of this protein. In addition, HBV further increased APIMI transcription resulting in significantly increased levels of this gene in the HepG2.215 cell line compared with those noted in HepG2 cells $(\mathrm{P}<0.001$; Fig. 1A). AP1M1 protein expression was further increased in liver cancer cells and was significantly increased following transfection with the HBV genome $(\mathrm{P}<0.05$; Fig. $1 \mathrm{~B}$ and $\mathrm{C})$.
To determine whether HBV infection could alter APIMI transcription in the liver, the expression profile data (GDS4387) of 27 samples from acute liver failure patients with or without HBV infection was analysed. The data were uploaded by Nissim et al (26). In agreement with the observations of the present study, HBV infection facilitated APIMI expression in the liver samples (Fig. 1D). Taken collectively, these data suggested that $A P I M 1$ was upregulated in liver cancer and that HBV infection could further facilitate its expression in liver cancer cells.

$H B V$ promotes proliferation of liver cancer cells through upregulation of APIM1. Numerous studies have confirmed that HBV infection and notably HBX expression, promotes hepatocellular carcinoma (HCC) aggravation by facilitating HCC cell proliferation and invasion (21-23). The exact mechanism of this process remains unclear (27-29). The contribution of the increased levels of AP1M1 that occur during HBV infection on the proliferation of liver cancer cells was investigated. APIMI expression was knocked down by siRNAs transfection. As presented in Fig. 2A and B, the expression levels of AP1M1 were reduced following administration with siRNAs, notably siRNA1 and therefore siRNA1 was used in the following study. The cell proliferation was subsequently tested by cell viability and colony formation assays. Silencing of APIMI could significantly $(\mathrm{P}<0.001)$ suppress the proliferative ability of HBV-transfected liver cancer cells (HepG2.215). This led to $\sim 25 \%$ cell growth reduction compared with the control cells (Fig. 2E and F).

To further dissect whether AP1M1 overexpression could promote liver cancer cell proliferation, the total APIMI expression cassette was inserted into the eukaryotic cell expression vector pcDNA3.1 and then transfected into HepG2 cells. The stable transfected HepG2-OEAP1M1 cells were established that exhibited AP1M1 overexpression (Fig. 2C and D). Concomitantly, the control cells HepG2-NC were also established (Fig. 2C and D). Cell viability and colony formation assays indicated that AP1M1 could significantly promote HepG2 proliferation $(\mathrm{P}<0.001)$. Compared with HepG2-NC, cell growth of HepG2-OEAP1M1 cells was increased $20 \%$ (Fig. 2E and F). Taken collectively, these findings suggested that AP1M1 was one of the crucial factors involved in the enhanced proliferation of liver cancer cells caused by HBV infection.

$H B X$ is the major functional protein in $H B V$ regulating APIMI expression. HBV possesses four overlapping open reading frames, namely $\mathrm{C}, \mathrm{P}, \mathrm{S}$ and $\mathrm{X}$ (24-26). The coding protein HBX was reported to possess multiple transcriptional regulatory activities $(2,30,31)$. The present study explored whether HBX alone could facilitate AP1M1 expression. In order to address this hypothesis, HBX overexpressing HepG2 cells (HepG2-HBX) and HBX deficient HepG2.215 cells (HepG2.215 $\triangle$ HBX) were constructed (Fig. 3A). While overexpression of HBX facilitated AP1M1 expression, HBX deficiency in HepG2.215 cells reduced AP1M1 protein levels (Fig. 3B). In accordance with the western blotting results, quantitative PCR indicated that transcription of APIM1 in HepG2-HBX cells was $\sim 1.5$ times increased compared with in HepG2 cells. The data indicated that the expression of AP1M1 
A

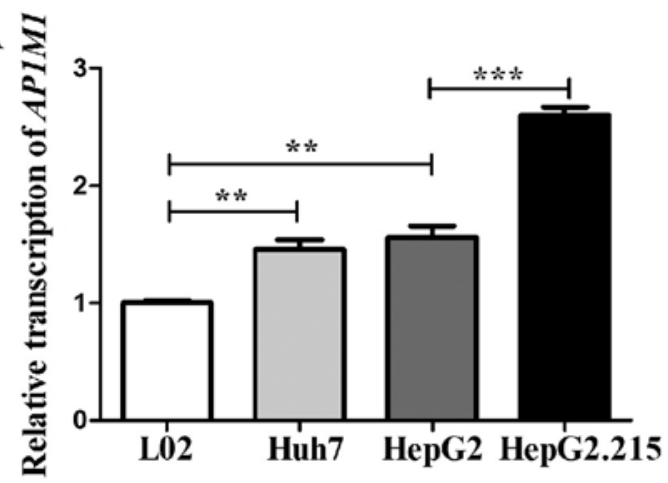

B

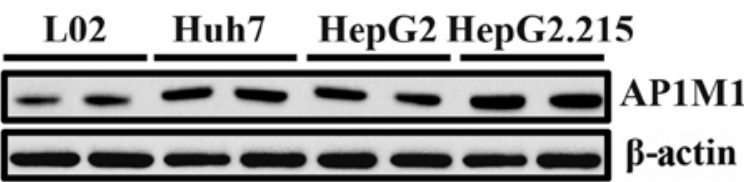

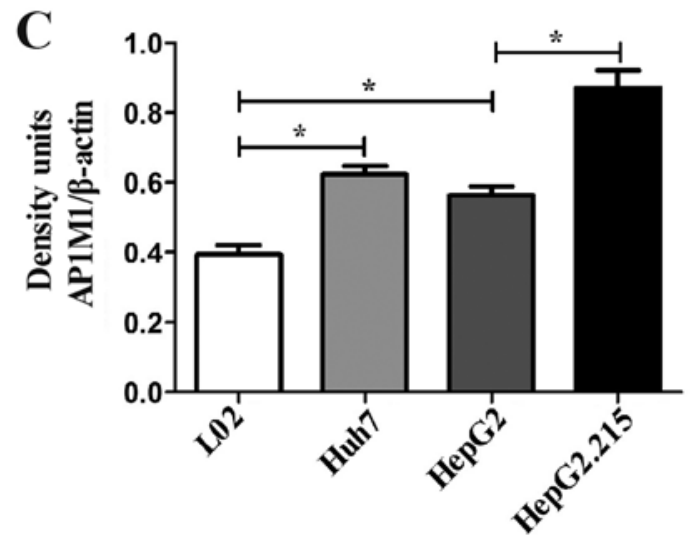

D

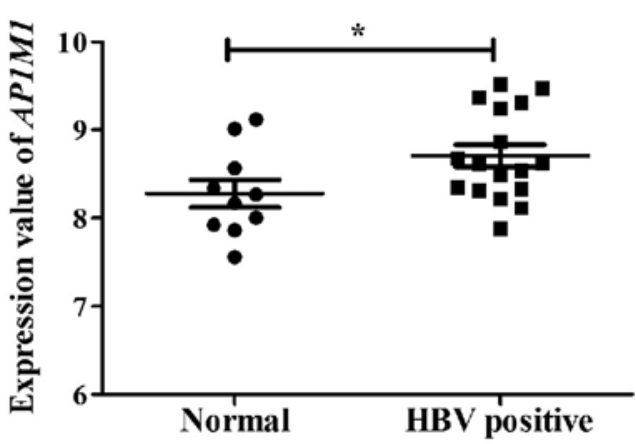

Figure 1. AP1M1 is upregulated in HBV-positive liver cancer cells. (A) Relative quantity of AP1M1 mRNA levels from L02, Huh7, HepG2 and HepG2.215 cells. (B) AP1M1 protein expression analysis was detected by western blotting in L02, Huh7, HepG2 and HepG2.215 cells. (C) The quantification of the proteins was performed by densitometry. (D) The relative transcription of AP1M1 in liver tissues of acute liver failure patients with or without HBV infection. ${ }^{*} \mathrm{P}<0.05,{ }^{* *} \mathrm{P}<0.01$ and ${ }^{* * *} \mathrm{P}<0.001$. HBV, hepatitis B virus.

was downregulated to $\sim 30 \%$ in HepG2.215 $\Delta$ HBX cells compared with that noted in HepG2.215 cells (Fig. 3C). The interaction of $\mathrm{HBX}$ with the $A P 1 M 1$ promoter by luciferase assays was further examined. The $A P 1 M 1$ promoter-driven coding sequence was transfected into liver cancer cells and the luciferase activity was determined as described in the Materials and methods section. HepG2-HBX and HepG2.215 cells demonstrated indiscriminate luciferase activity levels, while HepG2 and HepG2.215- $\triangle \mathrm{HBX}$ exhibited significantly lower activity levels $(\mathrm{P}<0.05)$ compared with HepG2-HBX and HepG2.215 cells (Fig. 3D). Taken collectively, the data indicated the role of HBX in facilitating AP1M1 expression in liver cancer cells.

However, the exact way by which the elevated expression of AP1M1 promotes liver cancer cell proliferation remains unknown. Yoneyama et al (21) demonstrated that AP1M1 binds to the YXXФ motifs of insulin receptor substrate (IRS-1) and subsequently activates the phosphotidylinositol 3 kinase (PI3K)-AKT pathway in order to induce cell proliferation in L6 myoblasts. Whether the expression of the human AP1M1 protein could also enhance PI3K-AKT activity and induce cell proliferation was examined. Total protein was extracted from HepG2, HepG2-OEAP1M1, HepG2.215 and HepG2.215-siAP1M1 cells and the phosphorylation of AKT was detected by western blotting. AP1M1 did not alter the amount of total AKT (Fig. 3E). However, the phosphorylation of AKT (Fig. 3E) was altered. Overexpression of AP1M1 increased the phosphorylation of AKT while its knockdown had the opposite effect. The data suggested that the human AP1M1 protein could activate the PI3K-AKT pathway. To investigate whether phosphorylation of AKT was required for liver cancer cell proliferation, the PI3K-AKT inhibitor LY294002 was administrated to HepG2-OEAP1M1 cells and a cell viability assay was performed. The data indicated that PI3K-AKT inhibition could significantly reduce cell viability $(\mathrm{P}<0.001)$. Moreover, the reduction effect was more profound in HepG2-OEAP1M1 cells compared with in HepG2-NC cells (Fig. 3F). This implied that the activation of the PI3K-AKT pathway was required for the regulation of liver cancer cell proliferation by AP1M1.

$H B V$ facilitates APIMI expression via the JNK pathway. Furthermore, the signaling pathways required for HBV and/or HBX regulation of AP1M1 transcription was investigated. It was reported that $\mathrm{HBV}$ or $\mathrm{HBX}$ alone could activate multiple signaling pathways, such as the NFKB, Wnt, PI3K-AKT and mitogen associated protein kinase (MAPK) pathways (32-35). To determine the key-signaling pathway, which regulated the expression of AP1M1, we initially conducted a prediction analysis of the binding sites of downstream transcription factors from representative pathways using online software (http://jaspar.binf.ku.dk/). As presented in Fig. 4A, the binding sites for NFKB1, c-Jun and c-Fos are located in the AP1M1 promoter region. Therefore, whether inhibition of the $\mathrm{NF} \kappa \mathrm{B}$ or the JNK pathways could downregulate the expression levels of AP1M1 was tested. The NFאB inhibitor BAY11-7082 did not 

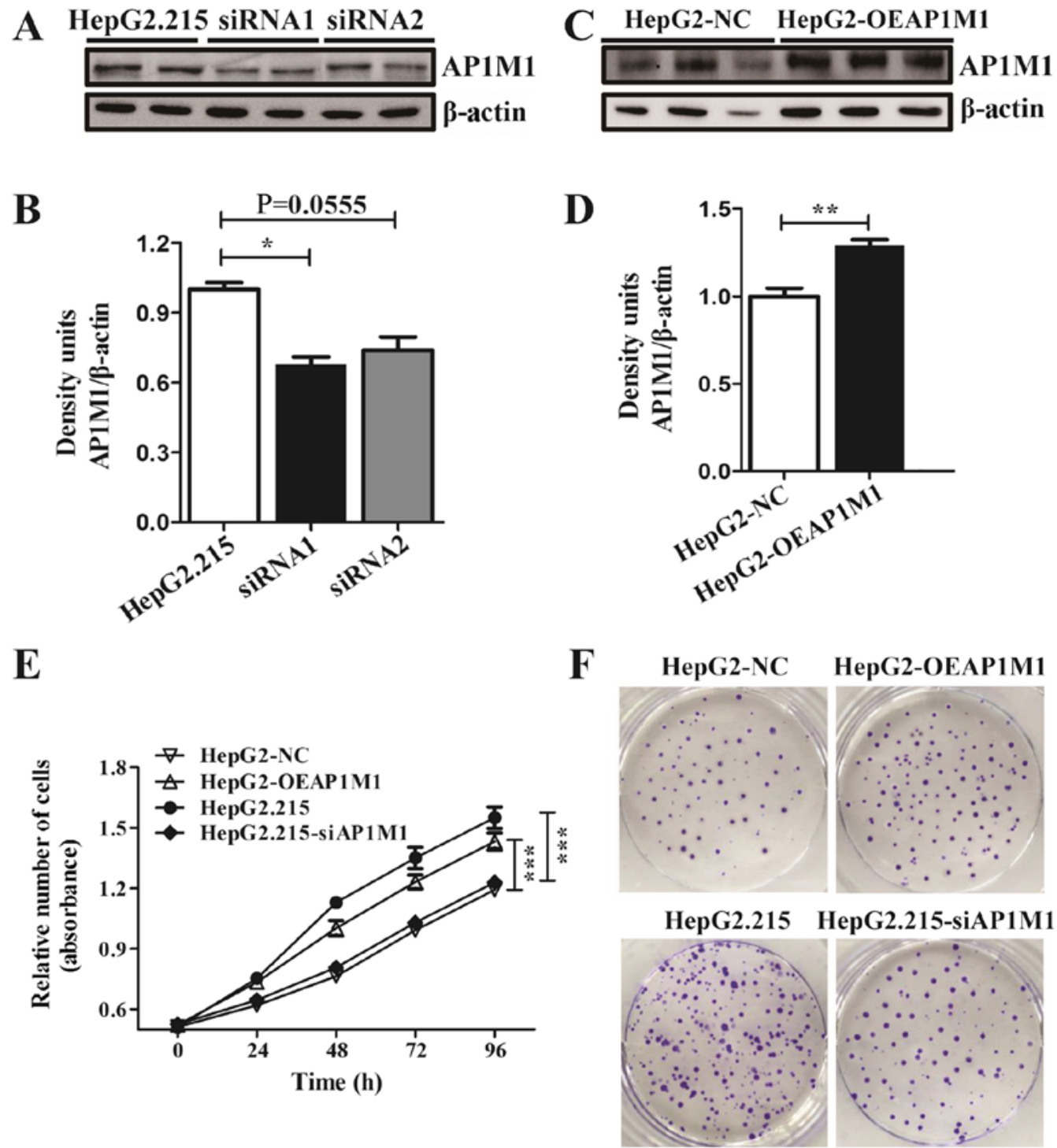

Figure 2. Upregulation of AP1M1 mediated by HBV promotes liver cancer cell proliferation. (A) Western blot analysis of AP1M1 from HepG2.215 cells with or without siRNA and (B) quantification of the proteins was performed by densitometry. (C) AP1M1 protein expression by western blot analysis in HepG2 cells overexpressing AP1M1 and control cells. (D) The quantification of the proteins was performed by densitometry. (E) Cell Counting Kit-8 and (F) colony formation assay analysis of cell proliferation among HepG2-NC, HepG2-OEAP1M1, HepG2.215 and HepG2.215-siAP1M1 cells. ${ }^{*} \mathrm{P}<0.05,{ }^{* *} \mathrm{P}<0.01$ and ${ }^{* * *} \mathrm{P}<0.001$. $\mathrm{HBV}$, hepatitis B virus; si, small interfering; NC, negative control.

suppress AP1M1 expression significantly (Fig. 4B). However, following inhibition of JNK phosphorylation, AP1M1 expression declined notably in HepG2.215 cells (Fig. 4C-E). These results were noted at the transcriptional (Fig. 4D) and protein level (Fig. 4C and E). Taken collectively, these data implied that JNK may be the key signaling protein involved in the HBV-mediated regulation of AP1M1 expression.

\section{Discussion}

Although previous studies have demonstrated that HBV infection can increase the risk of liver cancer, the exact molecular mechanism of this process remains undiscovered $(1,2,5,6)$. In the present study, it was demonstrated that AP1M1 acted as a cancer-promoting factor. AP1M1 overexpression promoted HepG2 proliferation, while its knockdown suppressed HepG2.215 proliferation. The data supported a model in which HBV infection or HBX expression upregulated AP1M1 expression by the JNK pathway. The abnormal expression of adaptin in turn promoted cell proliferation. Moreover, the results of the presented study indicated that the PI3K-AKT pathway was involved in this process and that overexpression of AP1M1 enhanced AKT phosphorylation in liver cancer cells. In contrast to AP1M1 overexpression, the silencing of AP1M1 inhibited AKT phosphorylation in the HBV-transfected liver cancer cells. These findings expand understanding of the function of adaptin in liver cancer.

In the past decades, cell biologists have unraveled the mechanism and specific function of adaptin (30-32). The canonical biological function of adaptin involves vesicular transport and protein sorting (36-38). Recently, novel biological roles of adaptin were identified, such as viral invasion, cell growth and hyperplasia. Using an in vitro cell culture system, it was demonstrated that the upregulation of human $\mu 1 \mathrm{~A}$ adaptin AP1M1 could promote liver cancer cell proliferation, which was in line with its homologous form reported 
A

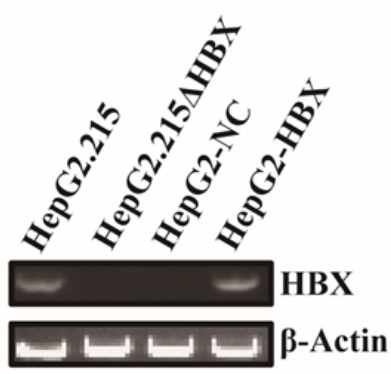

C

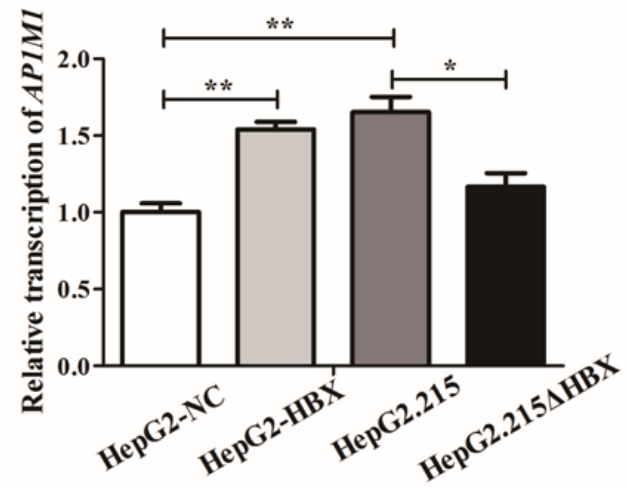

B

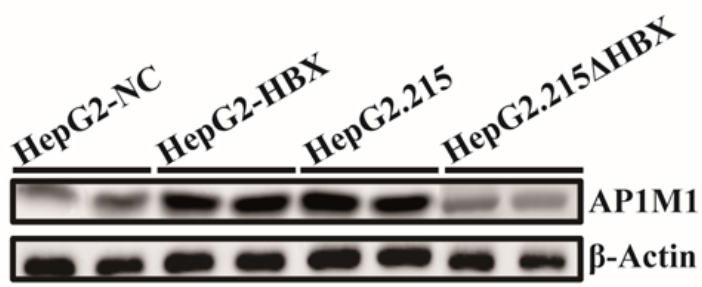

D

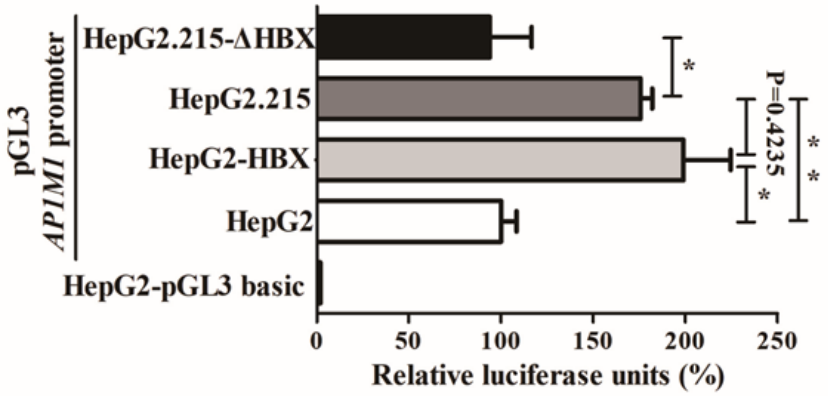

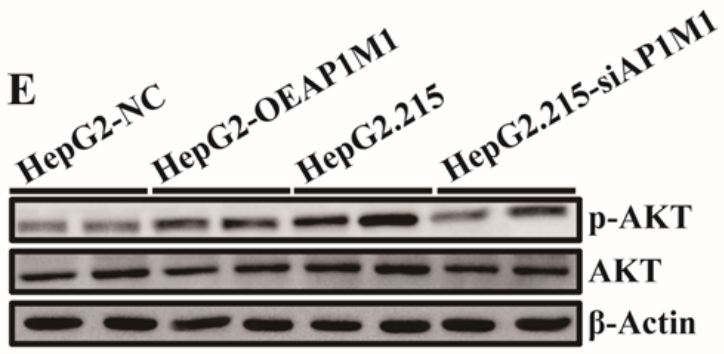

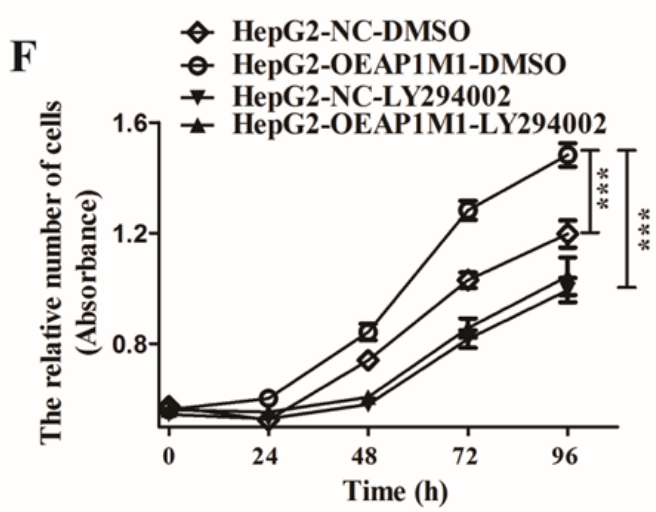

Figure 3. HBX facilitates AP1M1 expression in liver cancer cells. (A) HBX mRNA was detected by reverse transcription-PCR. (B) AP1M1 protein expression analysis by western blotting of HepG2-NC, HepG2-HBX, HepG2.215 and HepG2.215 $\mathrm{HBX}$ cells. (C) Quantitative PCR analysis of AP1M1 transcription in HepG2-NC, HepG2-HBX, HepG2.215 and HepG2.215 $\Delta$ HBX cells. (D) Dual luciferase assay analysis of AP1M1 promoter activity in HepG2-NC, HepG2-HBX, HepG2.215 and HepG2.215 $\Delta$ HBX cells. (E) Western blot analysis of total and phosphorylated AKT in HepG2-NC, HepG2-OEAP1M1, HepG2.215 and HepG2.215-siAP1M1 cells. (F) Cell Counting Kit-8 assay analysis of cell proliferation in HepG2 and HepG2.215 cells treated with or without LY294002. ${ }^{*} \mathrm{P}<0.05,{ }^{* *} \mathrm{P}<0.01$ and ${ }^{* * *} \mathrm{P}<0.001$. HBV, hepatitis B virus; p-AKT, phosphorylated protein kinase B; NC, negative control; si, small interfering.

in rats that regulated cell proliferation in L6 myoblasts (21). Hase et al (22) demonstrated the opposite function of the homologous form of AP1M1 in rodents. Specifically, it was demonstrated that adaptin $\mu 1 \mathrm{~B}$ deficiency in mice caused intestinal crypt hyperplasia with villous dysplasia owing to excessive proliferation of epithelial cells. The expression levels of $\mu 1 \mathrm{~B}$ adaptin AP1M2 were further examined in HepG2.215 and no differential expression was noted between HepG 2 and HepG2.215 cells regarding AP1M2 mRNA levels (data not shown). The data implied that only the expression of AP1M1 was regulated by $\mathrm{HBV}$ or $\mathrm{HBX}$. This process promoted cell proliferation in $\mathrm{HBV}$-positive liver cancer.

Current studies have demonstrated a variety of signaling pathways involved in HBV infection and liver cancer progression, including the NFאB, Wnt, PI3K-AKT, MAPK and Notch1 pathways (31-34). However, a limited number of studies have examined the interaction of these signaling pathways with AP1M1 or its homolog. Therefore, the key transcription factors involved in the signaling of the aforementioned pathways were initially investigated. Several NFאB1, c-Jun and c-Fos binding sites were predicted in the APIM1 promoter region. Subsequently, the pathway inhibitor was administered. Consistent with previous studies on other types of cancer cells $(39,40)$, the NFKB inhibitor BAY11-7082 suppressed proliferation of HepG2.215 and HepG2 cells, although no distinct difference was noted regarding AP1M1 levels. Given that JNK is one of the most important regulators of the AP-1 complex assembly by c-Jun and c-Fos (41), the effect of the JNK pathway on APIMI transcription was further tested and it was demonstrated that inhibition of JNK activity by SP600125 


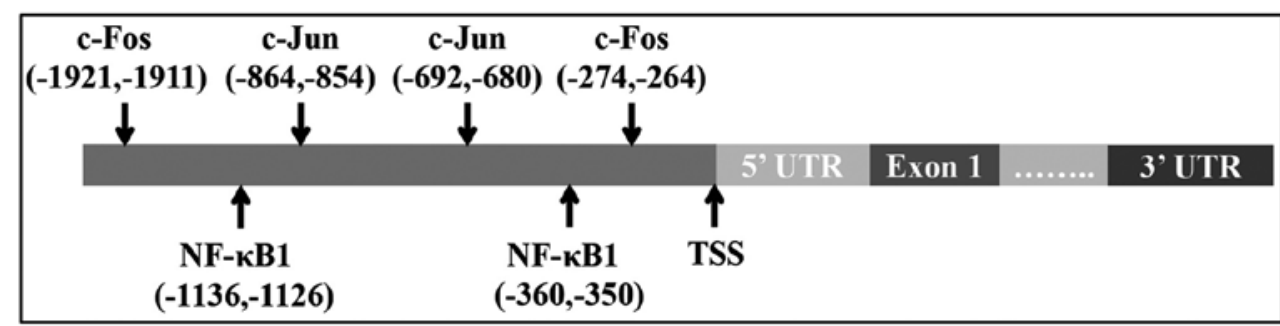

B
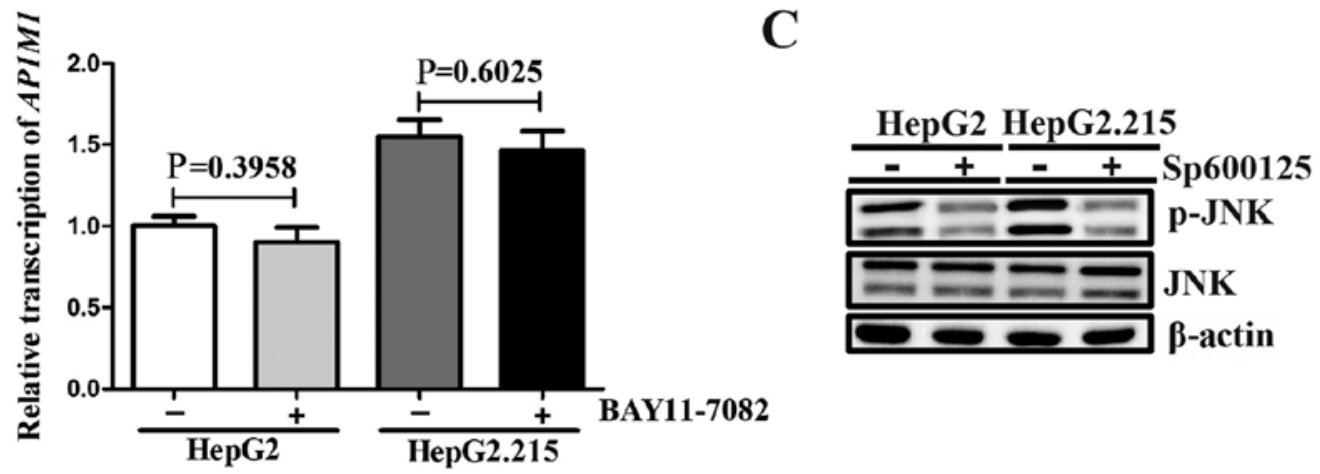

D

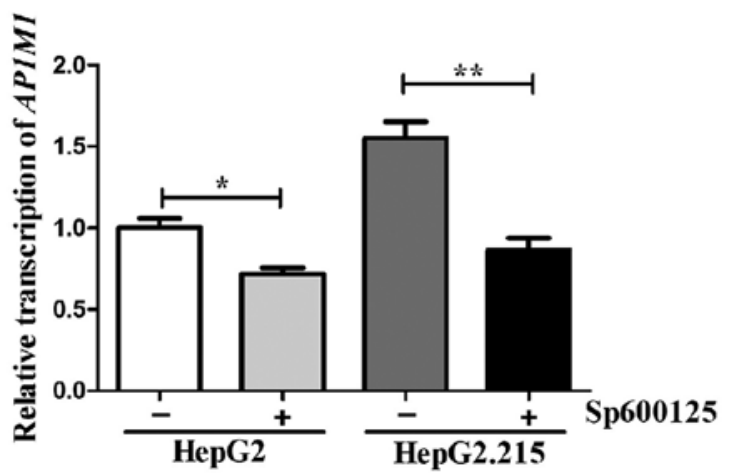

E

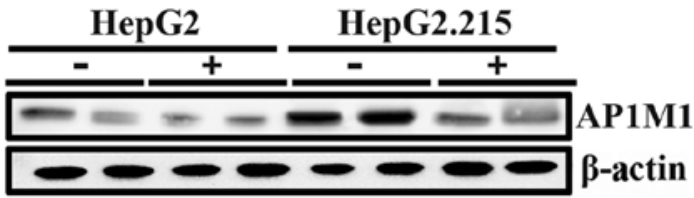

Figure 4. HBV upregulated AP1M1 expression in a JNK-dependent manner. (A) The transcription factor binding sites were scanned using the JASPAR online software. (B) Analysis of AP1M1 levels from HepG2 and HepG2.215 cells treated with BAY11-7082 by quantitative PCR. (C) Western blot analysis of total and phosphorylated JNK in HepG2 and HepG2.215 treated with or without Sp600125. (D) Quantitative PCR analysis of AP1M1 transcription in HepG2 and HepG2.215 cells treated with Sp600125. (E) AP1M1 protein expression analysis by western blotting. The samples were derived from HepG2 and HepG2.215 cells treated with or without Sp600125. ${ }^{*} \mathrm{P}<0.05$ and ${ }^{* *} \mathrm{P}<0.01$. NF, nuclear factor; HBV, hepatitis B virus; p-JNK, phosphorylated C-Jun N-terminal kinase; UTR, untranslated region.

downregulated AP1M1 mRNA and protein levels. It is possible that the regulation of AP1M1 expression is a complex process and that it is associated with multiple factors. JNK is possibly one of the key factors regulating HBV/HBX-induced AP1M1 expression. Whilst at the same time, JNK was reported to be activated by HBV/HBX and subsequently induced the proliferation of liver cancer cells $(5,42,43)$. However, the whole mechanism of JNK inducing liver cancer cells proliferation remains to be clarified. In the present study it was reported that one of the outcomes of activation of JNK pathway by $\mathrm{HBV} / \mathrm{HBX}$ was the upregulation of AP1M1 and the upregulation of AP1M1 promoted liver cancer cells proliferation.

Previous studies have suggested that $\mu 1$ adaptin impacts cell proliferation and growth in rodents and plants (20-22). Yoneyama et al further demonstrated that this function was associated with IRS-1 and that it subsequently activated the PI3K-AKT pathway in rat myoblasts (21). The present study confirmed this finding in human liver cancer cells that overexpressed AP1M1 and found that this increased expression activated the AKT protein. In contrast to this activation, the knockdown of AP1M1 inhibited AKT phosphorylation. Whether interaction of AP1M1 and IRS-1 accounts for the activation of PI3K-AKT in liver cancer cells is still unknown.

Taken together, the results suggest that HBX can promote AP1M1 expression via the JNK pathway. Moreover, the increased expression of AP1M1 accelerated liver cancer cell proliferation leading to liver cancer progression. The results presented in the current study were derived from in vitro experiments. Whether overexpression of AP1M1 promotes liver cancer proliferation in vivo is not clear. In addition, HepG2, which was the main cell line used in the present study has now been demonstrated to be derived from a hepatoblastoma origin. The incidence of hepatoblastoma has not been associated with HBV infection. However, the GeneChip data of the hepatocyte-derived carcinoma cell line Huh7 that have been reported from the authors' previous study (23), further indicated that transcription of $A P 1 M 1$ was upregulated in pUC18-HBV1.2 transfected Huh7 cells compared with the 
control Huh7 cells. In non-cancerous liver samples derived from acute liver failure patients, HBV infection facilitated AP1M1 transcription. Therefore, HBV infection can facilitate AP1M1 expression in liver cells. The results of the present study expand the understanding of the biological functions of adaptin in liver cancer. Further studies on the clinical application of AP1M1 may provide new therapeutic targets for HBV-positive liver cancer treatments.

\section{Acknowledgements}

Not applicable.

\section{Funding}

The present study was supported by the project of the National Natural Science Foundation of China (grant no. 81700774 to YK and grant no. 81501762 to WP), the Starting Foundation for Talents of the Xuzhou Medical University (grant no. 53591518 to $\mathrm{YK}$ ), the Priority Academic Program Development of Jiangsu Higher Education Institutions (PAPD) in the years of 2014 and 2018 (grant no. KYLX14-1448) and the Natural Science Foundation of the Jiangsu Higher Education Institutions (grant no. 16KJB310017 to FK).

\section{Availability of data and materials}

The datasets used and/or analyzed during the current study are available from the corresponding author on reasonable request.

\section{Authors' contributions}

YK and XY performed the western blotting, quantitative PCR and dual luciferase assays. QL, BZ and XL performed the cell viability and colony formation assays. XW, WP and FK constructed the plasmids. YW analyzed the GEO data. RT and $\mathrm{KZ}$ analyzed the raw data. All authors read and approved the final manuscript.

\section{Ethics approval and consent to participate}

Not applicable.

\section{Patient consent for publication}

Not applicable.

\section{Competing interests}

The authors declare no conflicts of interest.

\section{References}

1. El-Serag HB: Epidemiology of viral hepatitis and hepatocellular carcinoma. Gastroenterology 142: 1264-1273, 2012.

2. Kong F, You H, Tang R and Zheng K: The regulation of proteins associated with the cytoskeleton by hepatitis B virus $\mathrm{X}$ protein during hepatocarcinogenesis. Oncol Lett 13: 2514-2520, 2017.

3. Lo SJ, Lee Y, Chiou JS, Ting LP, Liu WT and Choo K: Characterization of restriction endonuclease maps of hepatitis B viral DNAs. Biochem Biophys Res Commun 129: 797-803, 1985.
4. $\mathrm{Xu} \mathrm{W}, \mathrm{Yu} \mathrm{J}$ and Wong $\mathrm{VW}$ : Mechanism and prediction of HCC development in HBV infection. Best Pract Res Clin Gastroenterol 31: 291-298, 2017.

5. Cho HK, Kim SY, Kyaw YY, Win AA, Koo SH, Kim HH and Cheong J: HBx induces the proliferation of hepatocellular carcinoma cells via AP1 over-expressed as a result of ER stress. Biochem J 466: 115-121, 2015.

6. Lin S and Zhang YJ: Interference of Apoptosis by Hepatitis B Virus. Viruses 9: pii: E230, 2017.

7. He Z, Yu Y, Nong Y, Du L, Liu C, Cao Y, Bai L and Tang H: Hepatitis $\mathrm{B}$ virus $\mathrm{X}$ protein promotes hepatocellular carcinoma invasion and metastasis via upregulating thioredoxin interacting protein. Oncol Lett 14: 1323-1332, 2017.

8. Geng M, Xin X, Bi LQ, Zhou LT and Liu XH: Molecular mechanism of hepatitis $B$ virus $X$ protein function in hepatocarcinogenesis. World J Gastroenterol 21: 10732-10738, 2015.

9. Yang ST, Yen CJ, Lai CH, Lin YJ, Chang KC, Lee JC, Liu YW, Chang-Liao PY, Hsu LS, Chang WC, et al: SUMOylated CPAP is required for IKK-mediated NF- $\mathrm{KB}$ activation and enhances HBx-induced NF- $\kappa B$ signaling in HCC. J Hepatol 58: 1157-1164, 2013.

10. Zhang F, Wang Q, Ye L, Feng Y and Zhang X: Hepatitis B virus $X$ protein upregulates expression of calpain small subunit 1 via nuclear factor-kappaB/p65 in hepatoma cells. J Med Virol 82: 920-928, 2010.

11. Doria M, Klein N, Lucito R and Schneider RJ: The hepatitis B virus $H B x$ protein is a dual specificity cytoplasmic activator of Ras and nuclear activator of transcription factors. EMBO J 14: 4747-4757, 1995.

12. Klein NP and Schneider RJ: Activation of Src family kinases by hepatitis B virus HBx protein and coupled signaling to Ras. Mol Cell Biol 17: 6427-6436, 1997.

13. Schmid SL: Clathrin-coated vesicle formation and protein sorting: An integrated process. Annu Rev Biochem 66: 511-548, 1997.

14. Doray B and Kornfeld S: Gamma subunit of the AP-1 adaptor complex binds clathrin: Implications for cooperative binding in coated vesicle assembly. Mol Biol Cell 12: 1925-1935, 2001.

15. Ohno H, Tomemori T, Nakatsu F, Okazaki Y, Aguilar RC, Foelsch H, Mellman I, Saito T, Shirasawa T and Bonifacino JS: Mu1B, a novel adaptor medium chain expressed in polarized epithelial cells. FEBS Lett 449: 215-220, 1999.

16. Navarro Negredo P, Edgar JR, Wrobel AG, Zaccai NR, Antrobus R, Owen DJ and Robinson MS: Contribution of the clathrin adaptor AP-1 subunit $\mu 1$ to acidic cluster protein sorting. J Cell Biol 216: 2927-2943, 2017.

17. Ohno H, Stewart J, Fournier MC, Bosshart H, Rhee I, Miyatake S, Saito T, Gallusser A, Kirchhausen T and Bonifacino JS: Interaction of tyrosine-based sorting signals with clathrin-associated proteins. Science 269: 1872-1875, 1995.

18. Mukherjee D, Hanna CB and Aguilar RC: Artificial neural network for the prediction of tyrosine-based sorting signal recognition by adaptor complexes. J Biomed Biotechnol 2012: 498031, 2012.

19. Tavares LA, da Silva EM, da Silva-Januário ME, Januário YC, de Cavalho JV, Czernisz ÉS, Mardones GA and daSilva LL: CD4 downregulation by the HIV-1 protein Nef reveals distinct roles for the $\gamma 1$ and $\gamma^{2}$ subunits of the AP-1 complex in protein trafficking. J Cell Sci 130: 429-443, 2017.

20. Park M, Song K, Reichardt I, Kim H, Mayer U, Stierhof YD, Hwang I and Jürgens G: Arabidopsis $\mu$-adaptin subunit AP1M of adaptor protein complex 1 mediates late secretory and vacuolar traffic and is required for growth. Proc Natl Acad Sci USA 110: 10318-10323, 2013.

21. Yoneyama Y, Matsuo M, Take K, Kabuta T, Chida K, Hakuno F and Takahashi S: The AP-1 complex regulates intracellular localization of insulin receptor substrate 1 , which is required for insulin-like growth factor I-dependent cell proliferation. Mol Cell Biol 33: 1991-2003, 2013.

22. Hase K, Nakatsu F, Ohmae M, Sugihara K, Shioda N, Takahashi D, Obata Y, Furusawa Y, Fujimura Y, Yamashita T, et al: AP-1B mediated protein sorting regulates polarity and proliferation of intestinal epithelial cells in mice. Gastroenterology 145: 625-635, 2013.

23. Kong F, Hu W, Zhou K, Wei X, Kou Y, You H, Zheng K and Tang R: Hepatitis B virus X protein promotes interleukin-7 receptor expression via NF- $\kappa \mathrm{B}$ and Notch1 pathway to facilitate proliferation and migration of hepatitis $\mathrm{B}$ virus-related hepatoma cells. J Exp Clin Cancer Res 35: 172, 2016. 
24. Tang R, Kong F, Hu L, You H, Zhang P, Du W and Zheng K: Role of hepatitis B virus X protein in regulating LIM and SH3 protein 1 (LASP-1) expression to mediate proliferation and migration of hepatoma cells. Virol J 9: 163, 2012.

25. Livak KJ and Schmittgen TD: Analysis of relative gene expression data using real-time quantitative PCR and the 2(-Delta Delta $\mathrm{C}(\mathrm{T})$ ) method. Methods 25: 402-408, 2001.

26. Nissim O, Melis M, Diaz G, Kleiner DE, Tice A, Fantola G, Zamboni F, Mishra L and Farci P: Liver regeneration signature in hepatitis B virus (HBV)-associated acute liver failure identified by gene expression profiling. PLoS One 7: e49611, 2012.

27. Hayashi S, Khan A, Simons BC, Homan C, Matsui T, Ogawa K, Kawashima K, Murakami S, Takahashi S, Isogawa M, et al: A novel association between core mutations in hepatitis B virus genotype F1b and hepatocellular carcinoma in Alaskan Native People. Hepatology 69: 19-33, 2019.

28. Waziry R, Grebely J, Amin J, Alavi M, Hajarizadeh B, George J, Matthews GV, Law M and Dore GJ: Survival following hospitalization with hepatocellular carcinoma among people notified with hepatitis B or C virus in Australia (2000-2014). Hepatol Commun 1: 736-747, 2017.

29. You H, Kong F, Zhou K, Wei X, Hu L, Hu W, Luo W, Kou Y, Liu X, Chen X, et al: HBX protein promotes LASP-1 expression through activation of c-Jun in human hepatoma cells. J Cell Physiol 233: 7279-7291, 2018.

30. Kong F, You H, Zhao J, Liu W, Hu L, Luo W, Hu W, Tang R and Zheng K: The enhanced expression of death receptor 5 (DR5) mediated by HBV X protein through NF-kappaB pathway is associated with cell apoptosis induced by (TNF- $\alpha$ related apoptosis inducing ligand) TRAIL in hepatoma cells. Virol J 12: 192, 2015.

31. Bak Y, Shin HJ, Bak IS, Yoon DY and Yu DY: Hepatitis B virus $\mathrm{X}$ promotes hepatocellular carcinoma development via nuclear protein 1 pathway. Biochem Biophys Res Commun 466: 676-681, 2015.

32. Kim SY, Kim JC, Kim JK, Kim HJ, Lee HM, Choi MS, Maeng PJ and Ahn JK: Hepatitis B virus X protein enhances NFkappaB activity through cooperating with VBP1. BMB Rep 41: 158-163, 2008.

33. Zheng BY, Gao WY, Huang XY, Lin LY, Fang XF, Chen ZX and Wang XZ: HBX promotes the proliferative ability of HL-7702 cells via the COX-2/Wnt/ $\beta$-catenin pathway. Mol Med Rep 17: $8432-8438,2018$.
34. Kongkavitoon P, Tangkijvanich $\mathrm{P}$, Hirankarn $\mathrm{N}$ and Palaga T: Hepatitis B virus HBx activates notch signaling via Delta-Like 4/Notch1 in hepatocellular carcinoma. PLoS One 11: e0146696, 2016.

35. Kang-Park S, Lee JH, Shin JH and Lee YI: Activation of the IGF-II gene by HBV-X protein requires PKC and p44/p42 map kinase signalings. Biochem Biophys Res Commun 283: 303-307, 2001.

36. Boehm M and Bonifacino JS: Genetic analyses of adaptin function from yeast to mammals. Gene 286: 175-186, 2002.

37. Robinson DG and Pimpl P: Clathrin and post-Golgi trafficking: A very complicated issue. Trends Plant Sci 19: 134-139, 2014

38. Robinson MS: Forty years of Clathrin-coated vesicles. Traffic 16 : $1210-1238,2015$

39. Zheng X, Chang RL, Cui XX, Avila G, Huang MT, Liu Y, Kong AN, Rabson AB and Conney AH: Inhibition of NF-kappaB by (E)3-[(4-methylphenyl)-sulfonyl]-2-propenenitrile (BAY11-7082; BAY) is associated with enhanced 12-O-tetradecanoylphorbol-13-acetate-induced growth suppression and apoptosis in human prostate cancer PC-3 cells. Int J Oncol 32: 257-264, 2008.

40. Bakhtiari E, Hosseini A, Boroushaki MT and Mousavi SH: Angiotensin II receptor antagonist olmesartan and NF-kappaB inhibitor as cytotoxic and apoptotic agents in MCF-7 human cell line. J Chemother 28: 314-320, 2016.

41. Uluçkan Ö, Guinea-Viniegra J, Jimenez M and Wagner EF: Signalling in inflammatory skin disease by AP-1 (Fos/Jun). Clin Exp Rheumatol 33 (4 Suppl 92): S44-S49, 2015.

42. Benn J, Su F, Doria M and Schneider RJ: Hepatitis B virus HBx protein induces transcription factor AP-1 by activation of extracellular signal-regulated and c-Jun N-terminal mitogen-activated protein kinases. J Virol 70: 4978-4985, 1996.

43. Natoli G, Avantaggiati ML, Chirillo P, Costanzo A, Artini M, Balsano $\mathrm{C}$ and Levrero $\mathrm{M}$ : Induction of the DNA-binding activity of c-jun/c-fos heterodimers by the hepatitis B virus transactivator pX. Mol Cell Biol 12: 989-998, 1994. International (CC BY-NC-ND 4.0) License. 